\title{
Modelling and characterization of a travelling-wave electro-optic modulator on InP
}

\author{
W. Pascher ${ }^{1}$, J. H. den Besten ${ }^{2}$, D. Caprioli ${ }^{2}$, R. van Dijk ${ }^{3}$, X. J. M. Leijtens ${ }^{2}$, and M. K. Smit ${ }^{2}$ \\ ${ }^{1}$ Allg. und Theoretische Elektrotechnik, FernUniversität, D-58084 Hagen, Germany \\ ${ }^{2}$ Opto-Electronic Devices group, Eindhoven University of Technology, Eindhoven, The Netherlands \\ ${ }^{3}$ TNO Physics and Electronics Laboratory, The Hague, The Netherlands
}

\begin{abstract}
A fast travelling-wave Mach-Zehnder modulator is modelled and designed using a rigorous vectorial analysis. In order to investigate propagation characteristics, velocity and microwave loss, the semiconductor layer stack and the lossy electrodes are modelled using the method of lines. The microwave field distribution is determined, design curves are derived and the cross-sectional dimensions of the modulator are optimized. The loss of the fabricated device agrees very well with small signal measurements up to $40 \mathrm{GHz}$ and HFSS simulations.
\end{abstract}

\section{Introduction}

High speed modulators are of great interest for $40 \mathrm{~Gb} / \mathrm{s}$ transmission systems and beyond. Travelling wave modulators (Walker, 1991) are not limited in frequency by the RC time constant. A Mach-Zehnder modulator has been realized, e.g., on GaAs (Walker, 1995) and on InP (Mörl et al., 1999; Krähenbühl, 1998). However, little is published about the microwave design considerations that are to be taken into account in the design of high-speed travelling wave MachZehnder modulators on InP. Highly accurate design data are necessary for these devices because of the long and costly fabrication process. Hence a rigorous model is needed that includes the main limiting factors: the velocity mismatch between the optical wave and the microwave as well as the microwave loss.

The waveguide structure of the modulator considered here (Fig. 2) is composed of several semiconductor layers with a step index profile and lossy metallic electrodes. Conventional analyses use a model with homogeneous dielectric layers and infinitely thin electrodes, which does not take the conductor losses into account. This yields an error in the microwave phase velocity and an incorrect electric field. In this paper, the model used fully accounts for the microwave and

Correspondence to: W. Pascher

(wilfried.pascher@FernUni-Hagen.de) optical properties of the inhomogeneous layer stack. The loss in the modulating electrodes and in the semiconductor layers is considered by means of a complex dielectric constant.

The model structure is analyzed by the method of lines $(\mathrm{MoL})$, which has been efficiently used for the simulation of various planar waveguides for microwave integrated circuits and integrated optics (Pregla and Pascher, 1989; Rogge and Pregla, 1993; Pregla and Pascher, 1995). The MoL takes advantage of the planar layer stack by discretizing the wave equation in the direction parallel to the interfaces of the layers, whereas an analytical solution is retained in the vertical direction. Thus highly accurate results are obtained at a comparatively small numerical effort. In this paper, we present MoL simulations to optimize the electric field across the reverse biased diode of the modulator. The results were confirmed by HFSS (High-Frequency Structure Simulator) and by measurements.

\subsection{Modulator layout and cross-section}

Since a reverse bias is used and almost no current is flowing in InP-based Mach-Zehnder switches, they can operate at sub-nanosecond speed. If lumped metal contacts are used, i.e. on top of the interferometer arms and on the bottom of the chip, the optical bandwidth of the modulator is limited to a couple of $\mathrm{GHz}$ by the capacitance. In a travelling wave design, the capacitance is distributed along the line and is consequently no longer a bandwidth limiting factor. In an ideal design, the modulating microwaves are travelling along the phase-shifter with a low attenuation at the same speed as that of the optical waves.

Figure 2 shows a cross-section of an interferometer arm. The RF-signal is fed to this modulator arm by a coplanar waveguide (CPW). At the beginning of the phase shifting sections, the CPW is transferred into a microstrip by a $\mathrm{n}^{+}$buffer layer underneath an optical ridge waveguide. In this ridge the light is confined in a $500 \mathrm{~nm}$ thick InGaAsP layer (Q1.25) with a bandgap wavelength of $1.25 \mu \mathrm{m}$. The InPcladding consists of an $200 \mathrm{~nm}$ thick intrinsic layer and a 


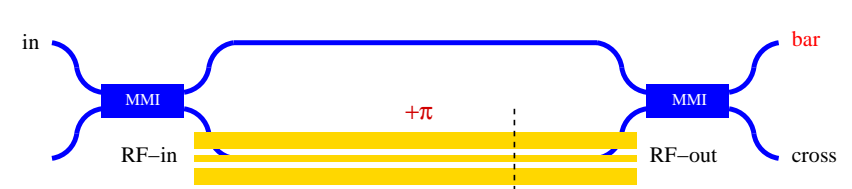

Fig. 1. Top view of a travelling-wave electro-optic modulator. The dashed line locates the cross-section of Fig. 2.

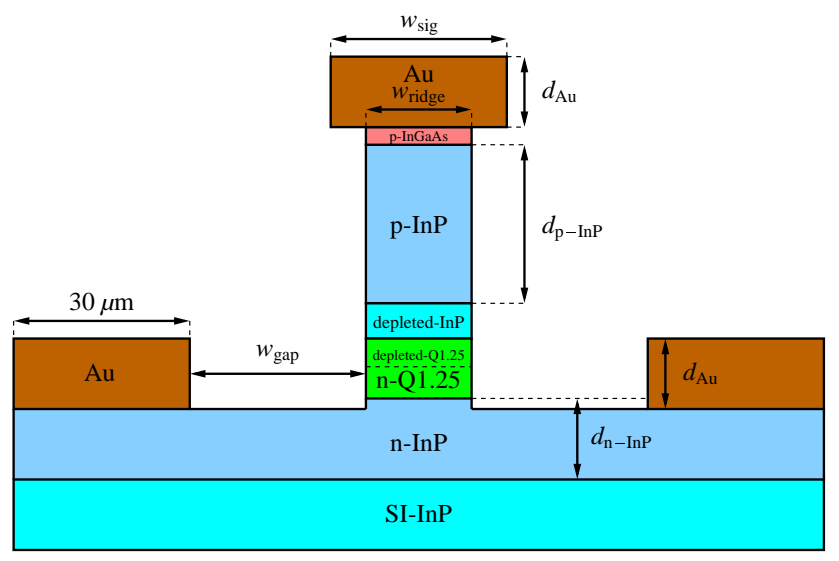

Fig. 2. Cross-section of a travelling-wave electro-optic modulator. The $\epsilon_{r}$ of InP/InGaAsP is 12.55 (the imaginary part is dependent on the doping level).

p-doped layer. Due to the pin-structure of the optical waveguide, the microwave electric field needed for the electrooptic effect will be across the depleted region, which consists of the upper part of the film layer and the intrinsic layer.

\section{Vectorial analysis by the method of lines}

The analysis is based on the method of lines (MoL) as presented in Pregla and Pascher (1989). The MoL is a semianalytic approach with analytic solution in one coordinate direction, namely perpendicular to the layers and a finitedifference discretization in the other direction. This approach is very well suited for multilayered waveguides as, e.g., for the travelling wave modulator under consideration. The cross-section of a modulator (as in Fig. 2) is partitioned into an "arbitrary" number of layers. These layers are inhomogeneous, also with permittivity steps $\left(\varepsilon_{r}=\varepsilon_{r}(x)\right)$, dielectric loss $\left(\varepsilon_{r}\right.$ complex) and metallic loss $\left(\varepsilon_{r}=-j \frac{\kappa}{\omega \varepsilon_{o}}\right)$.

The wave equations are established using two scalar potentials $\psi_{e}$ (electric) and $\psi_{h}$ (magnetic). We obtain a Helmholtz- and Sturm-Liouville differential equation for each layer

$$
\begin{aligned}
& \epsilon_{r}(x) \frac{\partial}{\partial x}\left(\frac{1}{\epsilon_{r}(x)} \frac{\partial \psi_{e}}{\partial x}\right)+\frac{\partial^{2} \psi_{e}}{\partial y^{2}} \\
& +\left(\epsilon_{r}(x) k_{0}^{2}-k_{z}^{2}\right) \psi_{e}=0
\end{aligned}
$$

$\frac{\partial^{2} \psi_{h}}{\partial x^{2}}+\frac{\partial^{2} \psi_{h}}{\partial y^{2}}+\left(\epsilon_{r}(x) k_{0}^{2}-k_{z}^{2}\right) \psi_{h}=0$

where we have assumed wave propagation in $z$ direction according to $\psi_{e, h} \sim \exp \left(-j k_{z} z\right)$.

The analysis steps of the method of lines for the electrooptic modulator are summarized in the flow chart (Fig. 3).

We apply a finite difference discretization in $x$ direction in order to convert to partial differential equations to coupled ordinary ones. In this way we are able to obtain an analytical solution in $y$ direction on discretization lines. Two sets of discretization lines are used, one for the electric potential $\psi_{e}$ and the other for the magnetic one $\psi_{h}$. The first derivative with respect to $x$ is expressed using finite differences and the following difference operator $\boldsymbol{D}$ for the magnetic potential $\psi_{h}$

$$
\begin{aligned}
& \left.\frac{\partial \psi_{h}}{\partial x}\right|_{(i+1 / 2) h} \approx \frac{\psi_{i+1}-\psi_{i}}{h} \text { or } \\
& h \frac{\partial \psi_{h}}{\partial x} \rightarrow \underbrace{\left[\begin{array}{rrrrr}
1 & & & & \\
-1 & 1 & & \\
& \ddots & \ddots & \\
& & \ddots & \ddots \\
& & & -1 & 1
\end{array}\right]}_{\boldsymbol{D}}\left[\begin{array}{l}
\psi_{1} \\
\psi_{2} \\
\vdots \\
\psi_{N-1} \\
\psi_{N}
\end{array}\right]=\mathbf{D} \psi_{h}
\end{aligned}
$$

The second derivative is given by

$h^{2} \frac{\partial^{2} \psi_{h}}{\partial x^{2}} \rightarrow-\boldsymbol{D}^{t} \boldsymbol{D} \boldsymbol{\psi}_{h}=-\boldsymbol{P}_{h} \boldsymbol{\psi}_{h}$

with tridiagonal $\boldsymbol{P}_{h}$ and analogous for the electric potential $\psi_{e}$. This yields the discretized wave equation (a system of coupled ordinary differential equations)

$\frac{d^{2}}{d y^{2}} \boldsymbol{\psi}_{h}-\left(k_{z}^{2} \boldsymbol{I}-\varepsilon_{h}+\boldsymbol{P}_{h}\right) \boldsymbol{\psi}_{h}=\mathbf{0}$

with a tridiagonal matrix $\mathbf{Q}_{h}$. In order to solve these equations, we perform a diagonalization, namely by transformation to principal axes:

$$
\begin{aligned}
& \boldsymbol{T}^{-1} \boldsymbol{Q} \boldsymbol{T}=\boldsymbol{\Gamma}^{2} \quad \text { (diagonal) } \\
& \boldsymbol{T}^{-1} \boldsymbol{\psi}=\overline{\boldsymbol{\psi}}
\end{aligned}
$$

This constitutes two separate eigenvalue problems for $\psi_{e}$ and $\psi_{h}$. We end up with the transformed Helmholtz equation

$\frac{d^{2}}{d y^{2}} \overline{\boldsymbol{\psi}}-\boldsymbol{\Gamma}^{2} \overline{\boldsymbol{\psi}}=\mathbf{0}$

which is decoupled and has an analytical solution in each individual layer

$\overline{\boldsymbol{\psi}}=\exp (-\boldsymbol{\Gamma} y) \mathbf{A}+\exp (\boldsymbol{\Gamma} y) \mathbf{B}$

In the following process the tangential fields are matched on each layer interface and we obtain a formulation of an indirect eigenvalue problem

$$
\begin{aligned}
\boldsymbol{Z}\left(\varepsilon_{r e}\right) \overline{\mathbf{H}}_{\text {tan }, M} & =\mathbf{0} \\
\operatorname{det}\left(\boldsymbol{Z}\left(\varepsilon_{r e}\right)\right) & =0
\end{aligned}
$$


2) FD Discretization
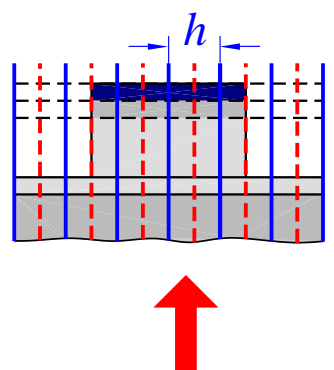

1) Partitioning of the cross-section

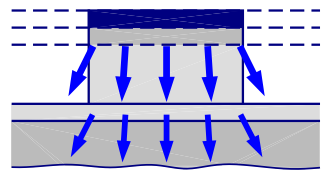

8) Field distribution
3) Transformation<smiles>[C]1CCCC1</smiles>

of the potential $\psi=\mathbf{T} \bar{\psi}$

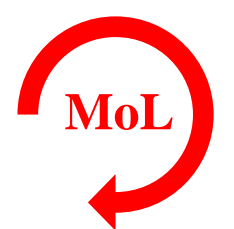

7) Inverse transform

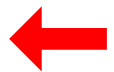

of the fields, e.g.,

$E_{x}=\boldsymbol{T}_{E} \bar{E}_{x}$
4) Analytical solution of the PDEs

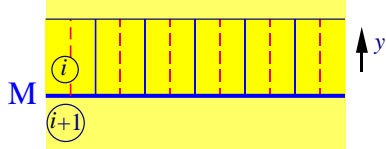

in one layer (i)

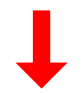

5) Field matching

on the interfaces $(\mathrm{M})$

$\rightarrow$ system equation

6) Numerical solution

$\rightarrow \gamma, \bar{E}, \bar{H}$

Fig. 3. Flow chart of the analysis steps in the MoL.

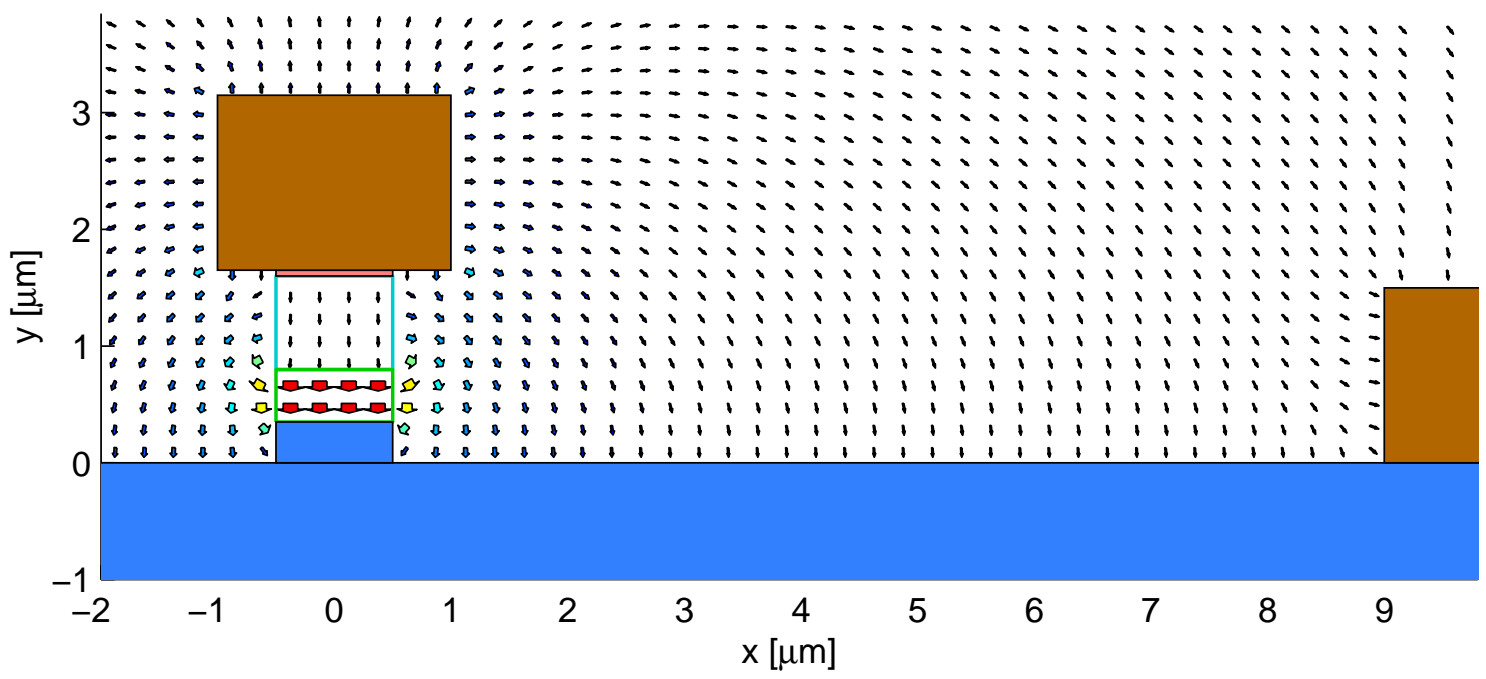

Fig. 4. Microwave electric field (arrow width proportional to field) in the cross-section of the optimized modulator.

which is solved for the the effective permittivity $\varepsilon_{r e}$ by a numerical search for zeros. This also gives the electromagnetic field $\overline{\mathbf{H}}, \overline{\mathbf{E}}$ in transform domain. Finally, an inverse transformation yields the field distribution in the waveguide.

\section{Simulation and measurement results}

The modulator has been simulated by the MoL according to the cross-section in Fig. 2. First the field distribution is given. Then design curves with respect to various dimensions of the device are presented in order to optimize its performance. Finally, the microwave loss and index are compared with small signal measurements and HFSS (High-Frequency Structure Simulator) simulations.

\subsection{Field distribution}

The microwave electrical field distribution is presented for the optimized design (see next section), namely in the right half of the cross-section (Fig. 4). It can be seen clearly that the microwave field is concentrated in the depletion region. The wide signal electrode, however, produces a stray field in air, which allows some control of the microwave refractive index. Obviously, the mode encountered is nearly a microstrip mode. The reason lies in the comparitively high conductivity of the n-doped buffer layer, which acts as a kind of ground plane. 

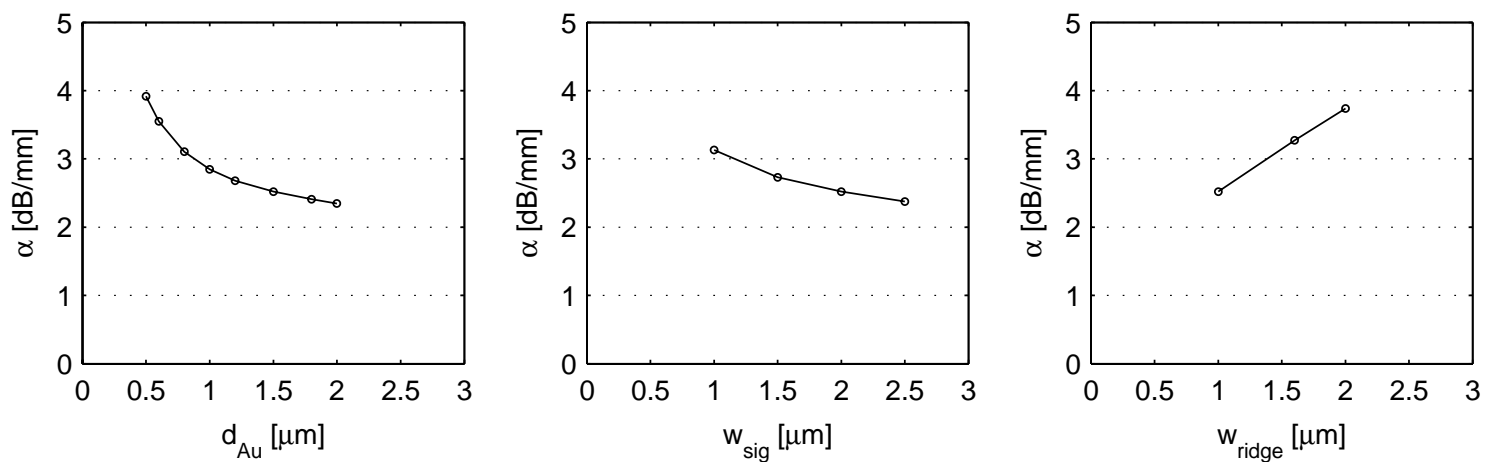

Fig. 5. Attenuation at $40 \mathrm{GHz}$ as a function of gold thickness thickness (left), signal line width (center), and p-cladding thickness (right). Other parameters are as in the optimized design.
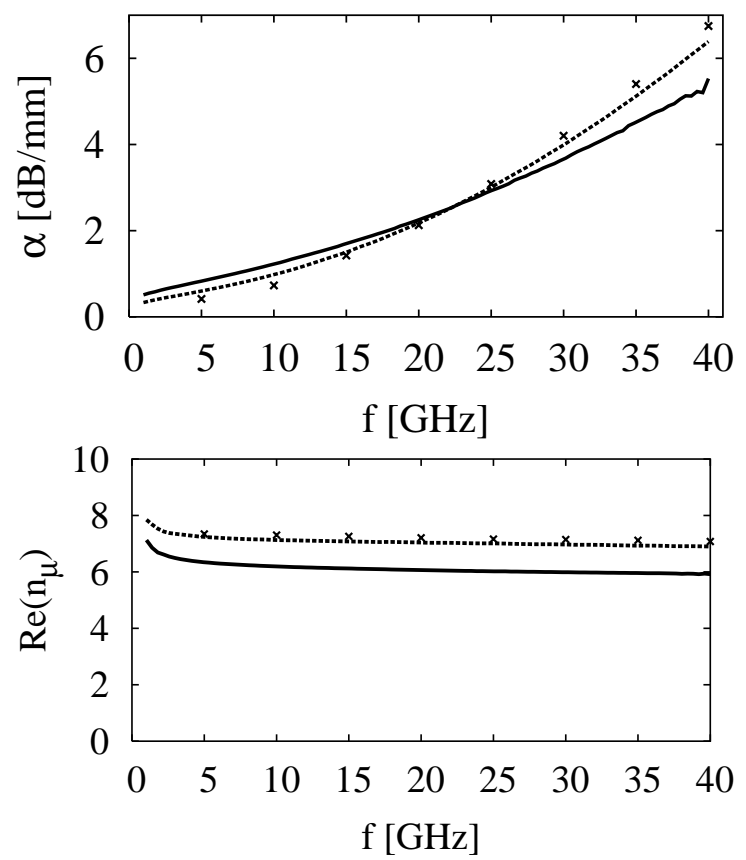

Fig. 6. Measurements (solid line), MoL-simulations (dashed line) and HFSS-simulations (crosses) of the microwave attenuation $\alpha$ (left) and the real part of the microwave index $n_{\mu}$ (right) for a phase shifter with a signal line width $w_{\text {sig }}$ of $12 \mu \mathrm{m}$.

\subsection{Design curves}

The biggest electro-optic effect will be obtained if the electric field is highest in the region where the light is confined. To make use of the pin-diode structure of the ridge, doping of the InP-ridge is necessary. Unfortunately, doped semiconductors have a conductivity that is much lower than that of metals. Therefore, the amount of doping in the cladding influences the RF-attenuation due to varying resistivity.

Figure 5 (simulated using the package MOLCAR; Kremer and Pregla, 1997) shows the attenuation is decreasing as the ridge width, and thus the amount of doped material in between the signal line and the depletion-zone edge, is de- creased. Figure 5 also shows the effect of the shape of the signal line on the RF-attenuation. A wider signal line width reduces the RF propagation loss, because a bigger part of the electric field is concentrated in the air instead of the semiconductor material. The attenuation is also reduced if the gold thickness is increased. Since the skin depth of Au is about $385 \mathrm{~nm}$ at $40 \mathrm{GHz}$, especially at thin Au thicknesses the attenuation increases dramatically. All plots were simulated at $40 \mathrm{GHz}$.

\subsection{Comparison with measurements}

The optimized design as described in the previous sections, has not been fabricated yet. Therefore, we checked measurements on already existing modulators with simulations by the Method of Lines. The left part of Fig. 6 shows the attenuation data of a structure with a ridge width of $4 \mu \mathrm{m}$ and a signal line width of $12 \mu \mathrm{m}$. The measured values for the phaseshifting section have been extracted using the thru-reflectline (TRL) method (Enge and Hoer, 1979). Depicted are the values measured through small signal (SS-)measurements, calculated by the MoL and calculated by HFSS.

HFSS simulations have been performed using the same structure as for the MOLCAR simulations. HFSS (Ansoft) is a 3-dimensional finite difference EM field solver. The structure is discretised in tetrahedra and the EM field components are calculated on the vertices and edges of these elements using appropriate boundary conditions. The mesh is refined adaptively at the locations where a large gradient exists. The output of a simulation is a.o. the impedance at the ports and the S-parameters. From these the complex propagation constant of the structure is calculated.

Comparison with measured values is very good. It can be noted that the loss curve does not follow a $\sqrt{f}$ law, since the losses are mainly determined by the absorption in the substrate and not by the skin depth of the electrodes.

The right part of Fig. 6 shows the real part of the microwave index of a structure with a signal line width of $12 \mu \mathrm{m}$. The high value of the index clearly shows that we are dealing with a slow wave structure which means that the velocity of the microwave is lower than the a wave in the 
materials considered. This is caused by the doped semiconductor layers.

One of the causes for the offset between simulation and measurement of the effective index could be the lack of reliable data of the used polyimide at RF-frequencies. Also, since the calculated values of the MoL and HFSS agree very well, we believe another source of error is the uncertainty of the doping levels in the different layers, which has not been measured. A different doping profile would not only change the conductivities, but also the thickness of the depletion layer. Attenuation and effective index plots for a structure with a signal line width of $w_{s i g}=6 \mu \mathrm{m}$ showed similar behaviour.

\section{Conclusion}

The method of lines (MoL) has been employed to model and design a fast travelling-wave electro-optic modulator. Field distributions and design curves have been presented and discussed in view of an optimized component with minimum microwave loss. The small-signal measurements of the fabricated device show very good agreement (better than $0.5 \mathrm{~dB} / \mathrm{mm}$ ) with the MoL modelling and with HFSS simulation results.

Acknowledgements. This work has been carried out with the partial funding of the European Union through the project IST 200025390 OBANET of the Information Society Technologies Research Programme and the Dutch NRC-Photonics programme.

\section{References}

Walker, R. G.: High-speed III-V semiconductor intensity modulators, IEEE J. Quantum Electron., 27, 3, pp. 654-667, Mar. 1991.

Walker, R. G.: GaAs/AlGaAs travelling-wave modulators for mmwave frequencies, IEE Colloquium Towards Terabit Transmission, pp. 4/1-4/6, 1995.

Mörl, L., Hoffmann, D., Matzen, K., Bornholdt, C., Mekonnen, G. G., and Reier,F.: Traveling wave electrodes for $50 \mathrm{GHz}$ operation of opto-electronic devices based on InP, Proc. IPRM99 conference, pp. 385-388, May 16-201999.

Krähenbühl, R.: Electro-Optic Space Switches in InGaAsP/InP for Optical Communication, Hartung-Gorre Verlag, Konstanz, second edition, 202 pp., 1998.

Pregla, R. and Pascher, W.: The Method of Lines, in T. Itoh (ed.): Numerical Techniques for Microwave and Millimeter Wave Passive Structures, New York, USA, J. Wiley Publ., pp. 381-446, 1989.

Rogge, U. and Pregla, R.: Method of Lines for the Analysis of Dielectric Waveguides, J. Lightwave Technol. 11(12), pp. 20152020, 1993.

Pregla, R. and Pascher, W.: Vectorial Analysis of Optical Waveguides by the Method of Lines, in Annual Review of Progress in Applied Computational Electromagnetics (ACES), 11, Monterey, USA, pp. 943-950, 1995.

Kremer, D. and Pregla, R.: MOLCAR a Very Efficient and Accurate Design Tool for the Hybrid Analysis of Multilayered Waveguide Structures, in Proc. SPIE Photonics West, Integrated Optics Devices: Potential for Commercialization, San Jose, USA, vol. 2997, pp. 255-262, Feb. 1997.

Enge, G. F. and Hoer, C. A.: Thru-reflect-line: an improved technique for calibrating the dual 6-port automatic network analyzer, IEEE Transactions on Microwave Theory and Techniques, 27, 12, pp. 987-993, Dec. 1979. 